\title{
Photon detection with CMOS sensors for fast imaging
}

\author{
J. Baudot, W. Dulinski, M. Winter \\ IPHC, Université Louis Pasteur, CNRS/IN2P3, BP 28, F-67037 Strasbourg, France \\ R. Barbier, E. Chabanat, P. Depasse, N. Estre \\ Université de Lyon, Université Lyon 1, Lyon, F-69003, France; Institut de Physique Nucléaire de Lyon, CNRS/IN2P3, Villeurbanne, \\ F-69622, France
}

\begin{abstract}
Pixel detectors employed in high energy physics, aim at detecting single minimum ionizing particle with micrometric positioning resolution. Monolithic CMOS sensors succeed in this task thanks to a low equivalent noise charge per pixel around 10 to $15 e^{-}$and a pixel pitch varying from 10 to a few 10s of microns. Additionally, due to the possibility for integration of some data treatment in the sensor itself, readout time of $100 \mu$ s have been reached for 100 kilo-pixels sensors.

These aspects of CMOS sensors are attractive for applications in photon imaging. For X-rays of a few keV, the efficiency is limited to a few $\%$ due to the thin sensitive volume. For visible photons, the back-thinned version of CMOS sensor is sensitive to low intensity sources, of a few hundreds of photons. But, when the back-thinned CMOS sensor is combined with a photo-cathode, the new hybrid detector resulting named EBCMOS, operates as a fast single photon imager. A first EBCMOS has been produced in 2007 and demonstrated single photon counting with low dark current capability in laboratory.

It has been compared in two different biological laboratories with existing CCD-based 2D cameras for fluorescence microscopy. The current EBCMOS sensitivity is comparable to existing EMCCD as well as its frame rate. On-going developments aim at increasing this rate by, at least, an order of magnitude. We report in conclusion, the first test of a new CMOS sensor, LUCY, which reaches 1000 frames/s.
\end{abstract}

\section{1. introduction}

CMOS sensors have been proposed [1] for standard lowcost cameras in the 1990 s. Taking advantage of the $100 \%$ fill factor and their low thickness, their development for high energy physics pixel detectors has started in 1999 at IPHC [2]. Indeed, vertex detectors for particle physics require granular, thin and swift sensors. Additional constraints come from radiation-tolerance and power consumption to limit the material budget and system complexity induced by cooling.

We consider in this contribution their interest for photon imaging. After a presentation of the general achievements of the MIMOSA sensors, we discuss the sensor ability to detect $\mathrm{X}$ rays of a few $\mathrm{keV}$. We then turn to the back-thinned version of CMOS sensors associated with a photo-cathode. This new hybrid photo-detector, called EBCMOS, provides single visible photon counting and positioning with high resolution at large frame rates. Results obtained with the first EBCMOS produced in 2007 are shown and its first operations in fluorescence microscopy for biology reported. We conclude on the current developments to reach
1000 frames per second and beyond.

The many prototypes of CMOS sensors from the MIMOSA series have demonstrated [3] the ability to detect single minimum ionizing particles (MIP) with an efficiency close to $100 \%$ while keeping a fake hit rate below $10^{-5}$ per pixel. The corresponding spatial resolution depends on the pixel size, from about $1 \mu \mathrm{m}$ for a $10 \mu \mathrm{m}$ pitch to $3 \mu \mathrm{m}$ for a $40 \mu \mathrm{m}$ pitch. These results are obtained with a sequential analog readout of each pixel of the matrix.

The key figure for these performances is the average noise per pixel. Indeed the equivalent noise charge ranges from 10 to $15 e^{-}$at room temperature, allowing for a signal over noise ratio for the pixel hit by a MIP above 15 (maximum probability value of the distribution).

Regarding the readout rate, the sequential analog readout results in a signal integration time per pixel of a few ms, equivalent to the readout time of the full matrix. In this strategy, parallelization of analog outputs limits the frame rate to the $\mathrm{kHz}$ for a mega-pixel detector.

In order to decrease the integration time, prototypes based 
on a column-parallel readout, where correlated double sampling is included in each pixel, have been developed [4]. In this mode, all the pixels of a given line are readout simultaneously, in 100 to $200 \mathrm{~ns}$, and their output value compared to a fixed threshold providing a binary readout. Hence, the integration time scales down to around $100 \mu \mathrm{s}$ for 1000 rows. The most recent Mimosa $22^{1}$ sensor, fabricated in 2007 , is made of a $576 \times 128$ pixels matrix read in $92 \mu$ s. Preliminary results from 2008 summer tests at the CERN-SPS exhibit an efficiency above $99 \%$ corresponding to a fake hit rate below $10^{-5}$ per pixel.

\section{Photon imaging possibilities}

The high speed single particle detection is an appealing feature for imagery. Indeed, it allows to estimate the position of individual particles, and their energy in some cases, providing more information than an integrated image. We investigate now, the sensitivity of CMOS sensors at different photon energies.

Since the sensitive volume is limited to the epitaxial layer, typically 10 to $20 \mu \mathrm{m}$, the efficiency for signals requiring large conversion volume is marginal. This is the case for gamma rays or X-rays above $10 \mathrm{keV}$ for which CMOS sensors are almost transparent.

Below $10 \mathrm{keV}$, X-rays will convert into the epitaxial layer with a $\%$ level efficiency. But when this conversion occur near to the charge collecting diode of the pixel, the full energy of the individual X-ray built up the signal of a single pixel. In this condition, the energy resolution reaches a few $\%$. This is illustrated by the signal distribution created by the soft X-rays at $5.9 \mathrm{keV}$ from a ${ }^{55} \mathrm{Fe}$ source in figure 1. The peak seen in one pixel corresponds to the $1640 e^{-}$generated by the X-rays and is fit with a Gaussian and an exponential to take into account the signal from pixels which have not collected all the charges generated. However due to the severe efficiency limitation, it is not clear which application may benefit from this energy resolution.

Visible light detection is obviously an application of standard CMOS sensor cameras. But, for much less intense sources, a back-thinned version of the sensor is required.

The industrial back-thinning process consist in removing the bulk part of the integrated circuit beneath the epitaxial layer. After a post-processing to passivate the surface, the sensitive volume is directly available for the so-called back-illumination.

A back-thinned version of the Mimosa 5 sensor has been obtained in 2003 through the SUCIMA FP5 European project [5]. Its ability to detect low energy (few keV) electrons have been thoroughly studied $[6,7]$. When backilluminated, such a sensor is able to detect a few hundreds visible photons arriving on a single pixel. Indeed they will

\footnotetext{
1 The Mimosa 22 chip has been designed by a common effort of the IPHC-Strasbourg and IRFU-Saclay groups.
}

Fig. 1. Signal from the conversion of soft X-rays from ${ }^{55} \mathrm{Fe}$ in the Mimosa 5 prototype. The peak appears at the end of the spectrum when all the generated charges are collected by one pixel.

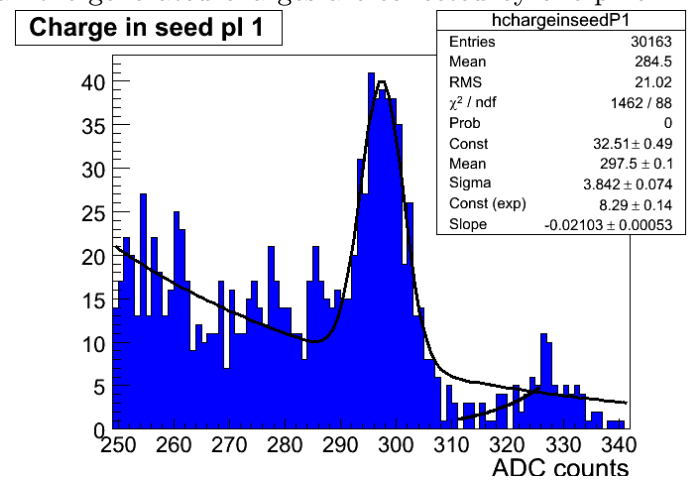

create around a few hundreds of charges which is a signal equivalent to the one produced by MIP. This detection mode could be interesting when the CMOS sensor is coupled to a crystal. Studies of such association is ongoing in our laboratories.

\section{Single photon sensitivity: the EBCMOS}

The ultimate photon sensitivity is reached when the back-thinned sensor is associated with a photo-cathode. The later converts a single photon to a photo-electron which, in turn, is accelerated in vacuum by an electrostatic field and bombards the back-side of the sensor. Such new hybrid photo-detectors are called EBCMOS for electrobombarded CMOS and its design is drawn on figure 2.

Fig. 2. Schematic design of the EBCMOS

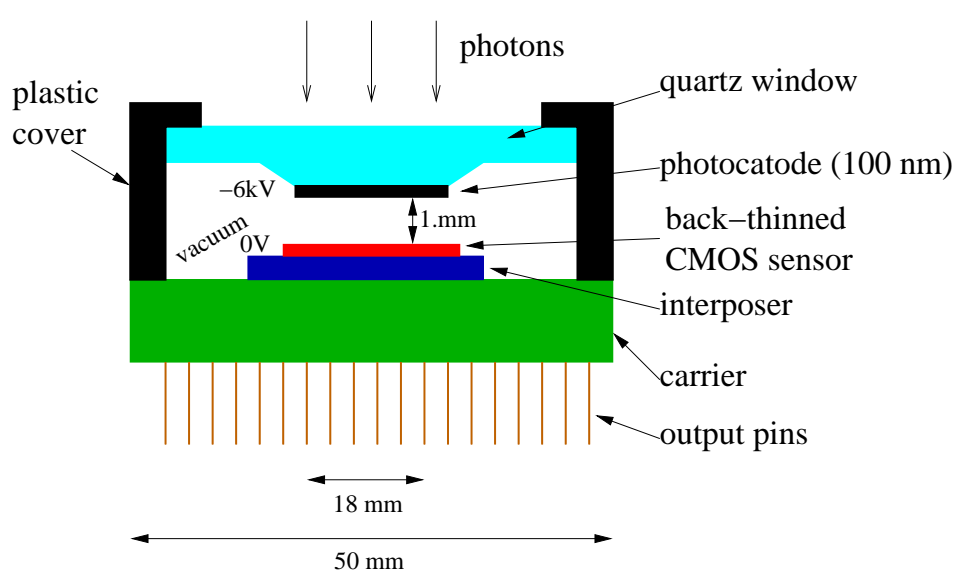

The gap and the electrostatic potential difference between the photo-cathode have to be carefully chosen. The high voltage has to provide enough acceleration for the electrons to cross the back-side passivation and penetrate the epitaxial layer. However, the voltage can not be arbitrarily high for obvious electrostatic reasons. Also, the dark current from the photo-cathode increases with the 
voltage. In turn, the spatial resolution is governed by the proximity focussing principle [8]. The resolution is expected to degrades linearly with the gap while improving with the square root of the voltage.

Based on the back-thinned Mimosa 5 sensor version, a first EBCMOS called EBMIMOSA 5 has been fabricated in 2007 with the Photonis company [9]. The photo-cathode used is of the S20 type, has a diameter of $18 \mathrm{~mm}$ and yields a quantum efficiency of $15 \%$ at $532 \mathrm{~nm}$. The photo-cathode to CMOS gap is $1 \mathrm{~mm}$ and the high voltage operation ranges from 6 to $10 \mathrm{kV}$. The Mimosa 5 sensor provides a $1024 \times 1024$ pixel matrix, with a pitch of $17 \mu \mathrm{m}$. The full matrix has an analog readout in $27 \mathrm{~ms}$ using a $10 \mathrm{MHz}$ clock, at which we currently operates, while the maximum frequency is $50 \mathrm{MHz}$.

EBMIMOSA 5 was thoroughly tested [10] with an optical bench where a calibrated laser diode light is focussed (1 $\mu \mathrm{m}$ diameter) on the photo-cathode. Both the single photon sensitivity and the photon counting capabilities were assessed. The detector exhibits a moderate dark count of $96 \mathrm{hits} / \mathrm{s} / \mathrm{mm}^{2}$ at a temperature of $10{ }^{\circ} \mathrm{C}$ below which it stays constant. The point spread function was establish to be $11.6 \pm 0.3 \mu \mathrm{m}$ at a wavelength of $540 \mathrm{~nm}$.

\section{First tests in biological environment}

Fluorescence microscopy as used in biology is one of the targeted applications of EBCMOS sensors. Biologists use fluorescent dyes to track down a given functionality of cells or molecules. An optical microscope allows to both excite fluorescent proteins and collect the emitted light. Building the image of interest requires a detector, which could be point-like or with 2D-positioning capability, depending on the microscope modality. Photo-multiplier tubes, used as point-like detector in confocal microscopy, are fast and single photon sensitive. Charge couple detectors offer 2Dpositioning but can not detect single photons. An evolution of CCDs, Electron Multiplying CCD, allows to reach the single photon sensitivity. Nevertheless, the CCD-based approach remains limited around 30 frames per second nowadays.

The newer microscope modalities [11], such as spinning disc microscope or PhotoActivated Localization Microscope (PALM) demand for the best sensitivity (single photon), 2D positioning and fast frame rate. It is clear that, improving by an order of magnitude the readout speed of $2 \mathrm{D}$ sensor while keeping the best sensitivity and high spatial resolution, will enhance microscope performances. The characteristics demonstrated by our EBCMOS in laboratory, should allow to fulfil this goal.

We have conducted the first tests [10] of EBMIMOSA 5 in epifluorescence setups in two biological laboratory to validate its principle. At IGBMC, Strasbourg [12], we studyed the sensitivity and contrast of our camera using cells from lilly roots whith low concentration of different fluorescent dyes (blue, green and red). For the green protein, see picture 3 , the constrast obtained was better than the commonly used CCD camera (CoolSNAP HQ2 from Roper Scientist) and comparable to an EMCCD (Cascade 2 from Roper Scientist) for the same integration time. We also imaged latex beads which diameter was below the microscope optical resolution power (typically $150 \mathrm{~nm}$ ). Accumulating images over 1 second and after temporal filter, we obtained the distribution of each bead localisation. The width of the latter, taken into account the magnicating factor of the microscope, reaches $245 \mathrm{~nm}$.

At ENS, Paris [13], the EBCMOS was set to observe membrane of hela cells including quantum dots. It has been possible to observe the blinking of a single point. This indicates that the camera was able to resolve a single quantum dots. These tests validated the use of EBCMOS sensors in fluorescence microscope with respect to the sensitivity (if the fluorescence dye emits light at wavelentgh compatible with the photocathode quantum efficiency) and spatial resolution. Our work focus now in enhancing the frame rate.

Fig. 3. Picture of cells from the lilly root obtlained with EBMIMOSA 5.

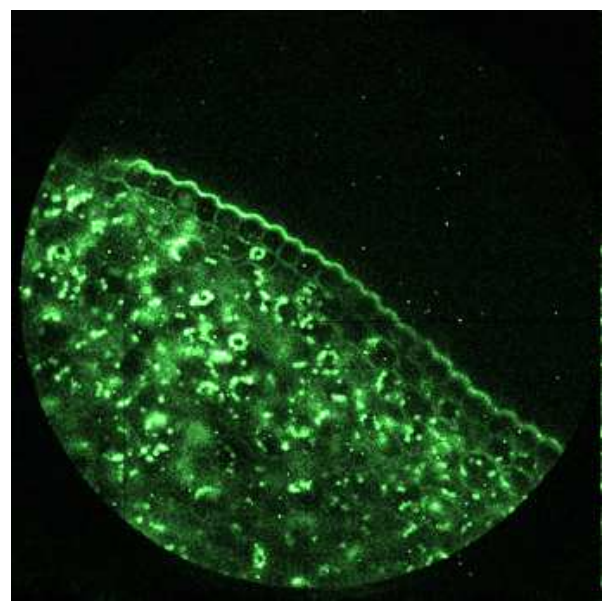

\section{On-going developments}

The first goal of on-going developments is to increase the frame rate which is currently limited to 40 frames per second with the EBMIMOSA 5 prototype. In 2007, a new chip, LUCY was designed and fabricated to reach 1000 frames per second. Based on a matrix of 400x800 pixels with a $10 \mu \mathrm{m}$ pitch, LUCY uses the analog readout strategy with 8 parallel outputs and a maximum frequency of $50 \mathrm{MHz}$. In laboratory tests using an ${ }^{55} \mathrm{Fe}$ source allowed to check the whole chip was functional and to estimate the equivlent noise charge per pixel between 8 to 10 e-. These performances are well suited for single particle detection which will be characterized soon by in-beam tests with MIP. The back-thinned version of Lucy is expected 
in September 2008 with low energy electron tests immediately following.

CMOS sensors with a large number of pixels and an analog readout set high constraints on the acquisition board digitizing the data prior to send them to a computer for image storage. Indeed, a mega-pixel sensor operating at 1000 frames per sec will generate a data rate around $8 \mathrm{Gbit} / \mathrm{s}$ ater an 8-bits digitization. A reduction of this enormous data throughput is possible if sparsification occurs in-line. We are conducting a board development including ADCs for parallel digitization of each outputs, on-board data treatment for inline hit reconstruction and Gbit/s output connection. Th board is ready for first tests with EBMIMOSA 5 and will be available for the operation of LUCY at the maximum frame rate.

Regarding future EBCMOS prototypes, the PHOTONIS group is investigating the possibility to use other photocathodes than the current S20 type. This is especially helpfull to enhance the quantum efficiency in a specific wavelength bandwith required by a given biology study. Another important work focus on reducing the gap between the photocathode and the back-plane of the CMOS sensor to improve the point spread function.

\section{Summary and conclusion}

The needs of high energy physics experiments have brought usual commercial CMOS sensors to the ability of detection single (charged) particle at high frame rate, above 1000 frames/s. Additionally, the small pixel pitch (down to $10 \mu \mathrm{m}$ ) available with the CMOS technology, leads to spatial resolution for such device at the micrometer level.

Imaging modalities which focus on tracking several sources at a fast rate and over a wide area, instead of integrating counts over this same area, may benefit greatly from this performances.

We have reviewed quickly the sensitivity of CMOS sensors to various photon wavelengths. The new hybrid photon detector associating a photocathode and a backthinned CMOS sensor, the EBCMOS, provides the first identifyed application for fluorescent microscopy. Indeed, in some cases, biologists use such microscopes with very low light intensity while requiring a high frame rate and 2D positioning.

We have validated the operation of our EBMIMOSA prototype, fabricated in 2007, on different image conditions in two biological laboratories. Its performances are today comparable to the most sensitive EMCCD camera. Further on-going developements will lead us in 2009 to overpass by at least an order of magnitude this current state-of-theart. Hopefully this will bring new discoveries in a field not connected from high energy physics from which this R\&D originated.

\section{Acknowledgements}

The authors whish to thanks all the members of the different groups which have provided the environment to produce the results presented here:

S. Vanzetto, G. Maurelli, R. Della Negra, C. Gurin, D. Bon, P-Y. Solane at the Institut de Physique Nucléaire in Lyon; A. Brognia, A. Besson, C. Colledani, R. De Masi, G. Doziere, C. Dritsa, A. Dorokhov, G. Clauss, JC. Fontaine, M. Goffe, A. Himmi, C. Hu, K. Jaaskelainen, M. Koziel, A. Merlin, F. Morel, N. Pillet, A. Shabetai at the Institut Pluridisciplinaire Hubert Curien in Strasbourg; H. Verboom, P. Clauzel, C. Kayser, N. Laurent at PHOTONIS-S.A. in Brives and Roden and also in the biology laboratories:

A. Giangrande, J.L. Vonesch, at IGBMC in Strasbourg; M. Dahan, I. Izeddin. at ENS in Paris.

\section{References}

[1] E. Fossum, IEEE Trans. Elec. Dev. 44, 1689 (1997).

[2] R. Turchetta et al., Nucl. Instr. Meth. A458, 677 (2001).

[3] M. Winter et al., DESY PRC report on R\&D N 01/04, (2007).

[4] Y.Li et al., Nucl. Instr. Meth. A572, 300 (2007).

[5] M. Caccia, et al., Nucl. Phys. B (Poc. Suppl.) 125, 133 (2003).

[6] G. Deptuch, Nucl. Instr. Meth. A543, 537 (2005).

[7] G. Deptuch, Ultramicr. 107, 674 (2006).

[8] A. Lyons, J. Phys. E: Sci. Instrum. 18 No 2, 127 (1985).

[9] R. Barbier et al., Proceedings of the ICATTP conference, 8-12 October 2007, Como, Italy.

[10] R. Barbier et al., Proceedings of the New Developments In Photodetection Conference, 15-20 June 2008, Aix-Les-Bains, France.

[11] S. Hell, Science 316, 1153 (2007).

[12] IGBMC stands for Institut de Gnomique et de Biologie Molculaire et Cellulaire. Biological samples and microscopes were provided by Angela Giangrande and Jean-Luc Vonesh.

[13] ENS stands for Ecole Normale Supérieure. Biological samples and microscopes were provided by Maxime Dahan. 